\title{
Training for Careers in Primary Care: Time for Attention to Culture
}

\author{
Denise M. Dupras, MD, $P h D^{7}$ and Colin P. West, MD, $P h D^{2,3}$ \\ 'Division of Primary Care Internal Medicine, Department of Medicine, Mayo Clinic, Rochester, MN, USA; ${ }^{2}$ Division of General Internal Medicine, \\ Department of Medicine, Mayo Clinic, Rochester, MN, USA; ${ }^{3}$ Division of Biomedical Statistics and Informatics, Department of Health Sciences \\ Research, Mayo Clinic, Rochester, MN, USA.
}

J Gen Intern Med 30(9):1243-4

DOI: $10.1007 / \mathrm{s} 11606-015-3416-1$

(c) Society of General Internal Medicine 2015

$\mathrm{T}$ here is general agreement that the primary care physician workforce is not sufficient to meet the evolving needs of our health care system. The aging population and the adoption of the Affordable Care Act will necessitate a more robust primary care system in the future. Estimates of the shortfall vary, but recent analyses suggest the need for at least 12,500 to 31,000 additional primary care physicians by the year $2025 .{ }^{1}$

Three papers in this Theme Issue of JGIM address the training of primary care physicians to meet this need. Kernan et al. ${ }^{2}$ suggest that medical student experiences in primary care should more effectively model ideal primary care practices. These experiences must not represent a false promise of what a student's future practice will be. Rather, Kernan and colleagues propose that these ideal training practices should benefit students, primary care physicians and institutions by stimulating broad change within primary care work environments in order to attract students and increase physician career satisfaction. To succeed, however, these practices will require a major cultural shift in the value placed on primary care at academic medical centers. This is not a trivial requirement: Kernan et al.'s core proposal to stimulate interest in primary care careers is to expose students to practices vastly different from what they currently have available to them within academic medical centers.

What evidence is available to guide these changes to our approach to training for primary care careers? Pfarrwaller et al. ${ }^{3}$ contribute a systematic review examining interventions published between 1993 and 2014 intended to encourage the selection of primary care specialties by medical students. This systematic review serves to build upon the prior work of Meurer et al. ${ }^{4}$ in asking three questions: which interventions can increase the proportion of students choosing a primary care specialty, what are the characteristics of successful interventions, and what is the quality of the literature supporting intervention recommendations? The authors identified 72 relevant studies, but unfortunately, most were at high risk of generating biased results. Subject to this limitation, the available evidence suggests that longitudinal programs

Published online July 15, 2015 emphasizing experiences in primary care or rural health are most likely to result in students choosing a career in primary care. The effect of other interventions is less clear. The primary message of this study is that more rigorous research with longterm outcomes is needed in order to understand which interventions offer the greatest benefit in producing primary care physicians. However, one key recommendation of both the prior study by Meurer et al. and the current study by Pfarrwaller and colleagues is that of addressing the need for a change in institutional culture to better support primary care careers.

Looking further down the training pipeline, Stanley et al. ${ }^{5}$ compared the output of primary care physicians over a 10 -year period for a categorical internal medicine track and two primary care internal medicine tracks at a single institution. Data on career outcomes and current practice were obtained from an alumni survey of graduates from 2001 to 2010. Outcomes from this study were remarkably similar to those found in a prior national study of career outcomes for categorical and primary care residency tracks. ${ }^{6}$ These findings are also very similar to prior studies that document both a reduction in the numbers of residents selecting careers as primary care physicians and a decreased interest in primary care during residency training, even among those who enter residency with a reported interest in primary care. ${ }^{7}$ Within the primary care tracks, $93 \%$ of graduates reported interest in primary care before entering residency, but only $54 \%$ reported practice in primary care after completing their residency. Although this is significantly greater than the $20 \%$ primary care practice rate among categorical residency graduates, it reflects sizable attrition in primary care over the course of training in tracks intended to produce primary care physicians and leaders.

The proportion of categorical residents in this study with an interest in a primary care career at the beginning of residency was low, at $23 \%$, but the reduction in the primary care career rate was actually far greater within the primary care tracks (from 93 to $54 \%$ ) than within the categorical track (from 23 to $20 \%$ ). Stanley and colleagues note that many residents reported that their ambulatory training experiences negatively influenced their career plans, although the specific adverse influences are not known. Regardless, the finding of a declining interest in primary care during training programs designed to develop primary care physicians suggests that expansive development of primary care residency training programs alone is likely inadequate to address the need for an expanded 
primary care physician workforce. Perhaps the loss of interest in primary care during residency could be stemmed or even reversed by attention to cultural shifts, as suggested by Kernan and colleagues, although Pfarrwaller and colleagues remind us that evidence for specific interventions is lacking.

So where do we go from here? There appear to be several critical intervention points for changing the composition of the physician workforce. Admitting students to medical school who are more likely to select a career in primary care may be one way to increase the future workforce pool. Factors associated with choosing a career in primary care include an interest in primary care upon entrance to medical school, interest in social issues and health disparities, volunteerism experiences, increasing age, and male gender. ${ }^{8}$ However, data suggest that many of these students will not practice primary care medicine unless their primary care experiences during medical school are radically altered. ${ }^{9,10}$ The review by Pfarrwaller et al. indicates how little we really know about how best to model primary care as a desirable career for medical students. This is an area ripe for investigation to better determine which interventions can meaningfully affect medical student career decisions. Finally, the article by Stanley and colleagues emphasizes the need to define the factors that discourage residents from entering primary care, and assess interventions to promote primary care as a desirable career for those closer to the completion of their training.

Clearly, there are myriad reasons that students and residents eventually select a particular career path, and there will be no simple solution to creating a primary care force able to meet the needs of our population. However, a common thread across these studies is the importance of changing the culture within academic medical centers. Without this fundamental change, it appears unlikely that interventions in medical school and residency will result in the primary care workforce we need. We propose that purposefully realigning the culture of academic medical centers - and indeed, medicine itself - to recognize the value of primary care and to elevate the stature of the primary care physician in support of society's primary care needs may, in fact, be the most important of the interventions required. What is necessary is a shift away from a culture with a "hidden curriculum" that disparages primary care as a career, toward an increased emphasis on education highlighting positive aspects of providing primary care. Within such a culture, medical students and residents would naturally gravitate to primary care careers whose value and meaning are highlighted rather than undermined by their training experiences. Changing the culture is a daunting task, but the health of our country depends upon it.

Conflict of Interest: All authors declare that they do not have a conflict of interest.

Corresponding Author: Denise M. Dupras, $M D, P h D$; Division of Primary Care Internal Medicine, Department of Medicine Mayo Clinic, 200 First Street SW, Rochester, MN 55905, USA (e-mail: dupras.denise@mayo.edu).

\section{REFERENCES}

1. The complexities of physician supply and demand: projections from 2013 to 2025. Association of American Medical Colleges, March 2015. Available at: https://www.aamc.org/download/426242/data/ihsreportdownload. pdf. Accessed 28 Apr 2015.

2. Kernan WN, Elnicki M, Hauer KE. The selling of primary care 2015. J Gen Intern Med. 2015 doi:10.1007/s11606-015-3364-9.

3. Pfarrwaller E, Sommer J, Chung $\mathbf{C}$, et al. Impact of interventions to increase the proportion of medical students choosing a primary care career: A systematic review. J Gen Intern Med. 2015 doi:10.1007/s11606015-3372-9.

4. Meurer LN, Bland CJ, Maldonado G. The state of the literature on primary care specialty choice: where do we go from here? Acad Med. 1996;71:68-77.

5. Stanley M, O'Brien $\mathbf{B}$, Julian $\mathbf{K}$, et al. Is training in a primary care internal medicine residency associated with a career in primary care medicine? J Gen Intern Med. 2015 doi:10.1007/s11606-015-3356-9.

6. West CP, Dupras DM. General medicine vs subspecialty career plans among internal medicine residents. JAMA. 2012;308:2241-7.

7. Jolly P, Erikson C, Garrison G. U.S. graduate medical education and physician specialty choice. Acad Med. 2013;88:468-74.

8. Specialty and geographic distribution of the physician workforce: what influences medical student and resident choices? American Academy of family Physicians: The Robert Graham Center, March 2009. Available at: http://www.graham-center.org/content/dam/rgc/documents/publications-reports/monographs-books/Specialty-geography-compressed.pdf. Accessed 28 Apr 2015.

9. Hauer KE, Durning SJ, Kernan WN, et al. Factors associated with medical students' career choices regarding internal medicine. JAMA. 2008;300: 1154-64.

10. Schwartz MD, Durning S, Linzer M, Hauer KE. Changes in medical students' views of internal medicine careers from 1990 to 2007. Arch Intern Med. 2011;171:744-9. 